\title{
On Some Aspects of the Structure-Property Relationships in SLM 3D-Printed Aluminum Alloy Parts
}

\author{
Pavel A. Somov ${ }^{1}$, Eugene S. Statnik ${ }^{1}$, Yuliya Malakhova ${ }^{2}$, Kirill V. Nyaza ${ }^{3}$, Alexey I. Salimon ${ }^{1}$, Dmitry Ryabov ${ }^{2}$ \\ and Alexander M. Korsunsky 4,1,*
}

1 HSM lab, Center for Energy Science and Technology, Skoltech, Moscow 121205, Russia; pavel.somov@skoltech.ru(P.A.S.); eugene.statnik@skoltech.ru(E.S.S.); a.salimon@skoltech.ru(A.I.S.)

2 Light Materials and Technologies Institute, UC RUSAL, Moscow 121096, Russia; yuliya.malakhova@rusal.com(Yu.M.), dmitriy.ryabov2@rusal.com(D.R.)

3 LLC "Rusatom - Additive Technology", Industrial Integrator of the SC Rosatom, Moscow 115409, Russia; mobiad@yandex.ru(K.V.N.)

4 MBLEM, Department of Engineering Science, University of Oxford, Oxford OX1 3PJ, United Kingdom

* Correspondence: a.korsusnky@skoltech.ru(A.M.K.)

\begin{abstract}
Recent years witnessed progressive broadening of the practical use of 3D-printed aluminium alloy parts, in particular for specific aerospace applications where weight saving is of great importance. Selective laser melting (SLM) is an intrinsically multi-parametric fabrication technology that offers multiple means of controlling mechanical properties (elastic moduli, yield strength, ductility) through the control over grains size, shape, and orientation. Ultimately, this approach implies that structural elements can be purposefully fabricated to reinforce specific zones and directions where higher mechanical loads are anticipated by design. Targeted control over mechanical properties is achieved through the tuning of 3D-printing parameters and may even obviate the need of heat treatment or mechanical post-processing. Systematic studies of grain structure for different printing orientation with the help of EBSD techniques in combination with mechanical testing at different dimensional levels are the necessary first steps to implement this agenda. Samples of 3Dprintable Al-Mg-Si RS-333 alloy were fabricated in 3 orientations with respect to the principal build direction and the fast laser beam scanning direction. Sample structure and proper-ties were investigated using a number of techniques, including EBSD, in situ SEM tensile testing, roughness measurements and nanoindentation. The as-printed samples we found to display strong variation in Young's modulus values from nanoindentation (from 43 to $66 \mathrm{GPa}$ ) and tensile tests (from 54 to 75 GPa), yield stress and ultimate tensile strength (100...195 and $130 \ldots 220 \mathrm{MPa})$ in different printing orientations, and almost constant hardness of about $0.8 \mathrm{GPa}$. A further preliminary study was conducted of the effect of surface finishing on the mechanical performance. Surface polishing appears to reduce Young's modulus and yield strength, but improves ductility, whereas the influence of sand blasting is more controversial. The experimental results are dis-cussed in connection with the grain morphology and orientation.
\end{abstract}

Keywords: RS-333 alloy; SLM 3DP; EBSD reconstruction; nanoindentation

\section{Introduction}

SLM 3D-printing of metallic materials is a technological process that initially emerged in the early 2000's as a route for rapid prototyping. Nowadays it has evolved into a mass-production method for the fabrication of highly demanding parts with complex shapes [1-3]. 3D-printable $\mathrm{Ti}, \mathrm{Ni}$ and $\mathrm{Al}$ alloys are progressively finding applications in the aerospace domain where weight saving issues are of great importance: gas turbine engine components [4, 5], fuselage structural elements [6], etc. The 3DP Al alloy RS-333 has been used recently for SLM fabrication of thermoregulatory casing of the gam-ma-ray detector mounted on the Yarilo satellite [6].

SLM 3D-printing is a flexibly controlled fabrication technique that is affected by the material composition and multiple process parameters (laser power density, scan speed, 
layer thickness, etc.) These disparate factors interact in a complex manner and lead to the emergence of various hierarchical microstructures with specific characteristics, e.g. arrangement and size of grains, grain boundaries, pores, and reinforcing phase particles. Numerous studies have been reported of the correlation between the fabrication parameters (laser wavelength and power, scanning rate and path width and overlap, growth direction with respect to the sample shape, design of supports, and many others), on the one hand, and internal structure and mechanical and functional properties for many metallic materials [7-10], and specifically for Al alloys [11-13]. Elongated columnar crystallites and equiaxed grains appear to be the pre-dominant structural elements in 3D-printed metals at the dimensional level of $10 . .300 \mu \mathrm{m}$ [14]. The more detailed particulars of the grain patterns (grain size, grain aspect ratio, and their crystallographic orientation with respect to the outer surfaces of the component) are likely to be governed by the local solidification conditions in the presence of directional heat flux and associated thermal gradients. It has also been noted that the mechanical properties such as elastic moduli and yield strength vary significantly depending on the relationship between the loading direction and the principal growth direction during printing, the fast scanning direction, etc.

We recently reported [13] a study of the mechanical performance of 3D-printed RS$333 \mathrm{Al}$-alloy parts and interpreted the variation of mechanical response in tension in terms of the different cooling conditions during printing (namely, the local time-temperature variation due to secondary re-heating of elementary volumes due to the deposition of subsequent layers above), which in turn affects the aging of the oversaturated solid solution obtained after printing. However, the grain pattern within the polycrystal aggregate was not studied in detail, although it was noted that the impact of this factor is likely to be significant.

In the present article we report a systematic EBSD study of the grain structure in 3D-printed RS-333 Al-Mg-Si alloy samples produced using different printing orientation and the establishment of a correlation with the results of mechanical property characterization using in-SEM tensile mechanical testing and nanoindentation. The strong influence is demonstrated of the grain assembly orientation with respect to the load direction on the overall mechanical performance. Additionally, the effect of sur-face finish quality is also investigated. We also discuss the possibility of grain growth control through accurate tuning of the thermal gradients and associated heat flux. The purposeful tuning of SLM process parameters in the course of a single printing process offers hitherto untapped opportunities for the fabrication of structural components with grain structure and properties that vary in the 3D component volume by design to deliver optimal mechanical performance.

When seen within a broad context, the approach explored in the current article belongs to the broad paradigm of optimal design for Additive Manufacturing. The underlying hypothesis of this approach is that achieving peak performance of the product produced by 3D-printing requires taking into consideration all relevant aspects, from alloy composition to process parameters to printing sequence and orientation. Addition-ally, the internal states of deformation and stress need to be taken into account, since they affect the overall sample mechanical response.

\section{Materials and Methods}

\subsection{Samples Preparation}

RS-333 (Al-3Si-0.5Mg) powder alloy supplied by Valcom-PM Ltd. (Volgograd, Russia) was used to SLM print flat dog-bone samples under argon atmosphere using an EOS M290 SLM printer (EOS GmbH, Maisach, Germany) equipped with a $400 \mathrm{~W}$ Yb-fibre laser, and gauge zone with the nominal length $\mathrm{x}$ width $\mathrm{x}$ thickness dimensions as $10 \times 4 \times 1 \mathrm{~mm}^{3}$, respectively. The details of printing and post-printing heat treatment procedures and sample geometry are given in our previous report [13]. 
Three sets of 3 samples had $Z X, X Z$ and $X Y$ orientations of main gauge axis in respect to the fast $(X)$ and slow $(Y)$ laser scanning directions and the growth axis $(Z)$. In our notation, the first character corresponds to the printing direction aligned along main gauge axis while the second is connected to the axis aligned with gauge width.

Printing supports used during printing were mechanically machined off to obtain samples of nominal dimensions. A set of standard ascending numbers of sandpaper and ultimately diamond paste were used for mechanical polishing of selected samples.

The sand-blasting treatment was carried out using $\mathrm{SiO}_{2}$ with a mesh of $+40 /-80$ microns. Average pressure of the air system was 7 bar, time for the sandblasting was 15 seconds for each surface.

In the Figure 1 general appearance of as-printed, polished and sand-blasted samples is illustrated. Substitutional difference of surface appearance is clearly seen and, surprisingly, some coarsening of surface clusters is present and smashed features appear after sand blasting.
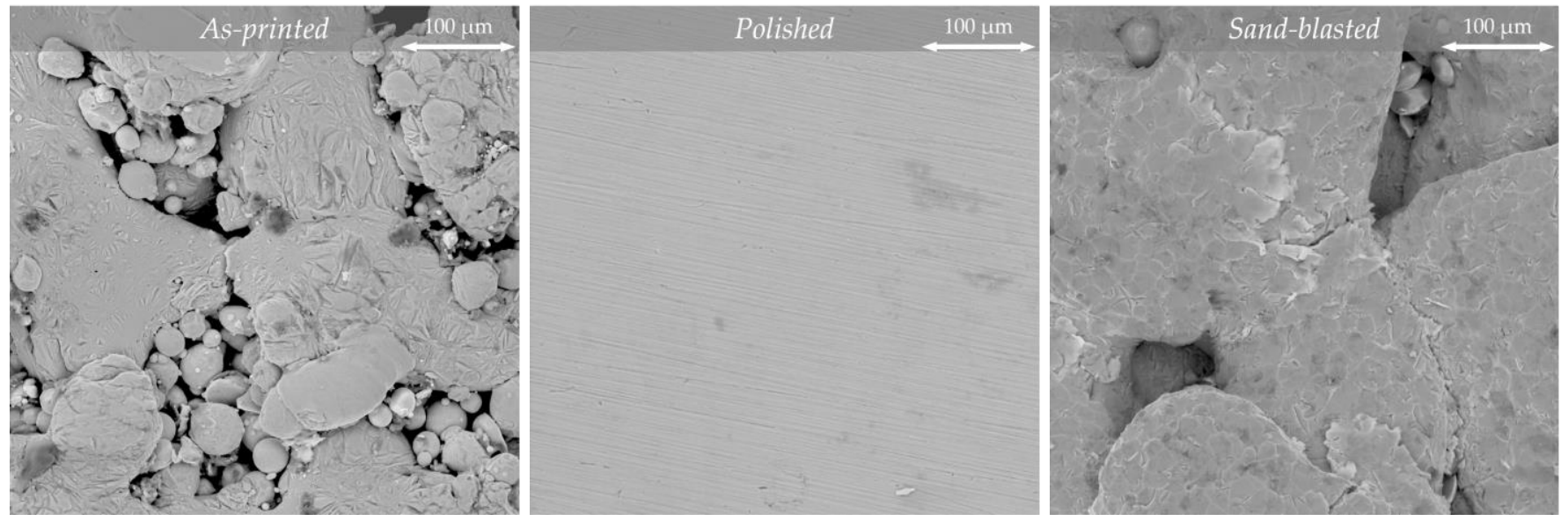

Figure 1. General appearance of as-printed, polished and sand-blasted samples.

\subsection{Electron Back Scatter Diffraction (EBSD)}

Since EBSD and nanoindentation measurements are very dependent on the surface quality and the morphology, samples must be carefully prepared before investigation. Each sample was cut out, next embedded in epoxy resin, and polished using standard metallographic techniques. Polishing was performed using sandpaper in several steps (220, 500, 1000, 2000, 4000 grit), then using diamond suspensions (average particles diameter 1-3 $\mu \mathrm{m}$ ) and colloidal silica. The final step of polishing was broad beam ion milling performed in a Technoorg Linda SEMPrep2 (Technoorg Linda Co. Ltd., Budapest, Hungary) using $8 \mathrm{kV}$ for 15 minutes with the $4^{\circ}$ stage tilt. After a final cleaning, the mounted sample was fixed in a metallographic mount holder to avoid image drift during the scanning process.

The surface quality was checked by optical profilometer of NanoScan4D system (FSBI TISNCM, Troitsk, Russia). The roughness was $346 \mathrm{~nm}$ that considers acceptable for future measurements. The general view of prepared surface with corresponding roughness profile is shown in Figure 2.

Texture was measured in a Scanning Electron Microscope (SEM) Tescan Vega3 (Tescan Orsay Holding, Brno, Czech Republic), using Oxford Instruments NordlysNano EBSD detector (Oxford Instruments NanoAnalysis \& Asylum Research, High Wycombe, UK). Measuring area was $500 \mu \mathrm{m}^{2}$ with step size $1 \mu \mathrm{m}$, acceleration voltage $20 \mathrm{kV}$, and beam current $10 \mathrm{nA}$. 

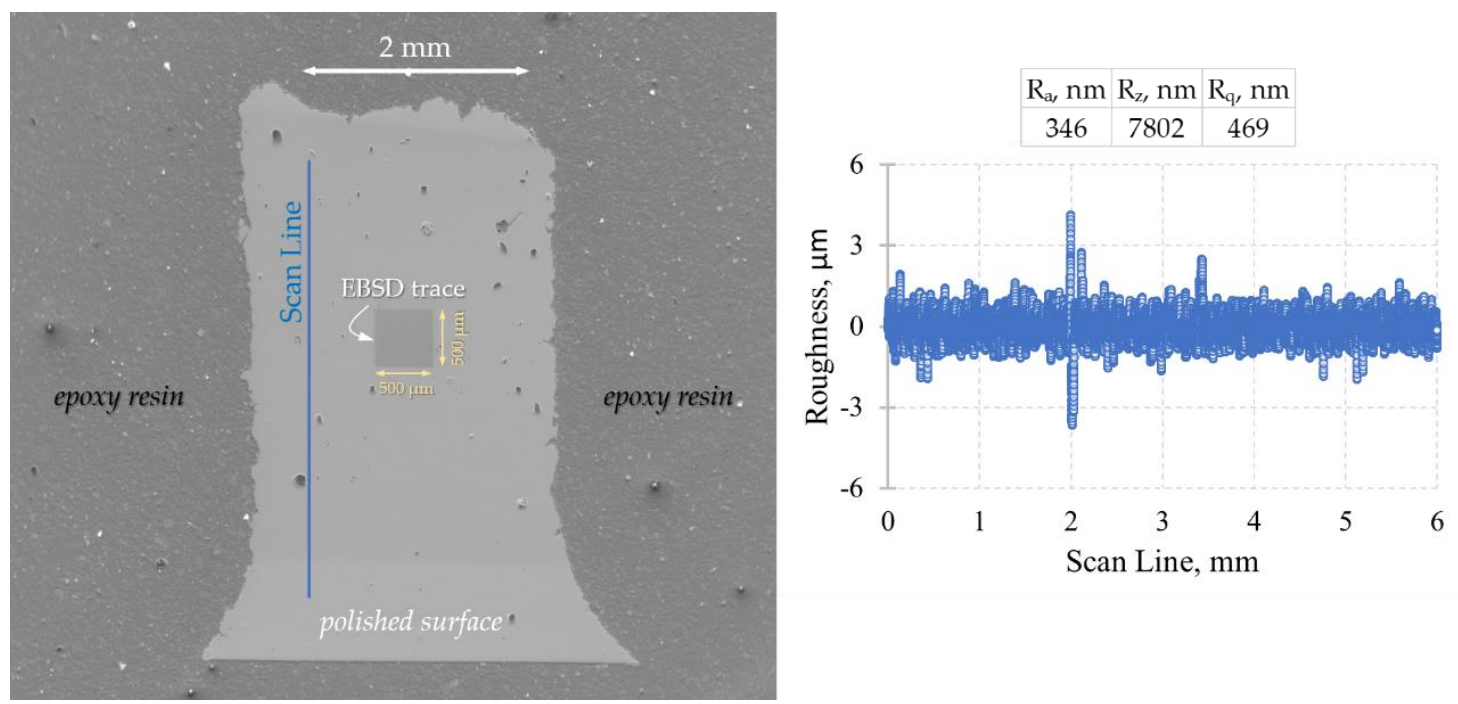

Figure 2. General view of polished surface for EBSD and nanoindentation measurements.

\subsection{Nanoindentation}

Nanoindentation test was performed for each orientation of polished specimens that were called as Cross-section (perpendicular to main gauge axis, width $\mathrm{x}$ thickness, $4 \times 1$ $\mathrm{mm} \times \mathrm{mm}$ ), Side (parallel to main gauge axis, length $\times$ thickness, $10 \times 1 \mathrm{~mm} \times \mathrm{mm}$ ), and Surface sections (parallel to main gauge axis, length $\mathrm{x}$ width, $10 \times 4 \mathrm{~mm} \times \mathrm{mm}$ ), respectively. The grid was 10x5 indents with X-step $200 \mu \mathrm{m}$ and Y-step $75 \mu \mathrm{m}$ along grains growth direction. The indentation parameters were the following: depth-control mode, penetration depth $1.5 \mu \mathrm{m}$, load time $10 \mathrm{~s}$, hold time $60 \mathrm{~s}$ and free unloading.

The post-processing technique was carried out according to the typical Oliver and Pharr methodology [15]. However, the achieved results showed high elastic modulus values ( 100 GPa) and hardness ( 2 GPa). It is well-known that Oliver and Pharr's model does not consider the impact of "heaps" (surplus material after indentation) formed around the indent. Therefore, the $\mathrm{E} / \mathrm{H}$ values for highly ductile materials may differ from those obtained with a tensile testing machine or a microindentation test. In this case, "highly ductile" roughly means a large E/H ratio (more than $\sim 20$ ) and refers to a small index of hardening.

In order improve this model and obtain reasonable values, there are at least two approaches. The first one [16] connects the discrepancy with the presence of plastic re-gion on the unloading curve of aluminum. The authors corrected this phenomenon with the repeated loading and achieved an elastic modulus of $\sim 70 \mathrm{GPa}$ for the pure aluminum plate. In another article [17], the authors proposed calculating the work under the indentation curve and make corrections.

In this article, we chose the second approach based on the work-of-indentation correction. The reduced elastic modulus was calculated via the formula

where $W_{e}$ is elastic work;

$$
\frac{W_{e}}{W_{e}+W_{p}}=\kappa \cdot \frac{4 \beta^{2} \gamma^{2}}{\pi} \cdot \frac{F_{\max }}{S^{2}} \cdot E_{R}
$$

$W_{p}$ is plastic work;

$\kappa=5.17$ when $\frac{W_{e}}{W_{e}+W_{p}}>0.25$ and $\kappa=7.3$ when $\frac{W_{e}}{W_{e}+W_{p}}<0.15$;

$\beta=1.05$ is coefficient taken from FEA analysis;

$\gamma=1.064$ is only Poisson's coefficient dependent;

$F_{\text {max }}$ is maximum load under the test;

$S$ is stiffness;

$E_{R}$ is reduced elastic modulus. 
Since parameters $W_{e}, W_{p}, F_{\text {max }}$, and $S$ can be taken from experimental data (indentation curves), the reduced elastic modulus was determined. The material elastic modulus was recalculated according to the formula

$$
\frac{1}{E_{R}}=\frac{1-v^{2}}{E}+\frac{1-v_{i}^{2}}{E_{i}}
$$

where $E, v$ are elastic modulus and Poisson's ratio of investigated material;

$E_{i}, v_{i}$ are indenter parameters (device passport data).

The relationship between hardness and reduced elastic modulus was expressed like the formula below. It is based on numerical simulations under a perfectly rigid indenter and instrument assumption.

$$
H=\frac{4 \beta^{2} \gamma^{2}}{\pi} \cdot \frac{F_{\max }}{S^{2}} \cdot E_{R}^{2}
$$

The above formula is attractive since it allows the hardness calculations without the determination of the contact area, which often requires the calibration of the indentation test to take into account the influence of the tip defect.

\subsection{Tensile Test}

In SEM mechanical testing is a growing experimental technique based on in situ and operando principles. A Deben Microtest 1-kN testing stage (Deben UK Ltd., Woolpit, UK) was accommodated the chamber of a Tescan Vega 3 SEM and connected with power and information cable to a computer-controlled operation block using a plug inserted to a through-flange plug. This facilitates the operation of testing stage that synchronises the mechanical loading (tension at permanent crosshead speed of $0.2 \mathrm{~mm} / \mathrm{min}$ ) with the acquisition of SEM images at the rate of $22 \mathrm{~s}$ per image in the secondary electron (SE) regime using $30 \mathrm{kV}$ voltage and beam spot size of $400 \mathrm{~nm} \mathrm{[13].}$

Digital image correlation (DIC) analysis was applied to retrieve the data on true strains in the region of interest $\sim 80 \%$ of gauge zone. The DIC algorithm searches for the corresponding pairs of pixel subsets (arrays containing digitalised intensity values) to find the best match in two digital images. When the centre positions of corresponding pixel subsets are identified, the displacement and strains (after the differentiation of displacements) can be calculated. The open-source Matlab-based software Ncorr [18] was utilized to analyse the datasets of acquired SEM images and to calculate average values of displacement and strain with subpixel resolution in the region of interest.

\section{Results and Discussion}

\subsection{Grain pattern appearance in 3D-printed $R S-333$ alloy}

As represented in Figures 3, 4 and 5 EBSD microscopy qualitatively reveals main motives of grain pattern appearance in 3D-printed RS-333 alloy depending on the printing orientation. It seems that grains have almost random crystallographic orientation against principal axes of a sample (no or weak texture), but the sizes and aspects of grains are obviously correlating with printing orientation - main axes of slim elongated columnar grains are always aligned along growth direction, and as a result, these grains appear as equiaxial in the plane normal to growth direction. In the planes parallel to growth direction elongated columnar grains are not single elements of grain pattern - round-like regions (spots) containing very small equiaxial grains and pores are likely evenly and randomly represented in these planes being interwoven with columnar grains. These spots are also readily noticeable at the plane normal to growth direction.

It is also worth to note that columnar grains may form almost parallel or divergent bouquet-like patterns in the planes parallel to growth direction. These and other peculiarities of grain patterns (size, aspects and crystallographic orientation statistics) are, however, to be thoroughly quantitatively analyzed at bigger subsets to validate a number of 
conceptual models which can be put forward to describe grain pattern formation, that will be addressed by authors together with FEM modelling of heat fluxes in a separate paper.

One can also notice that $X Y$ printing orientation returns somewhat different grain pattern than $\mathrm{ZX}$ and $\mathrm{XZ}$ printing orientations: thinner and shorter columnar grains, larger number of spots in the planes parallel to growth direction and coarser equiaxial grains in the plane perpendicular to growth direction. This is supposed to be directly related with specific remelting and solidification conditions - longest exposure time and diffused heat flow from pin supports.
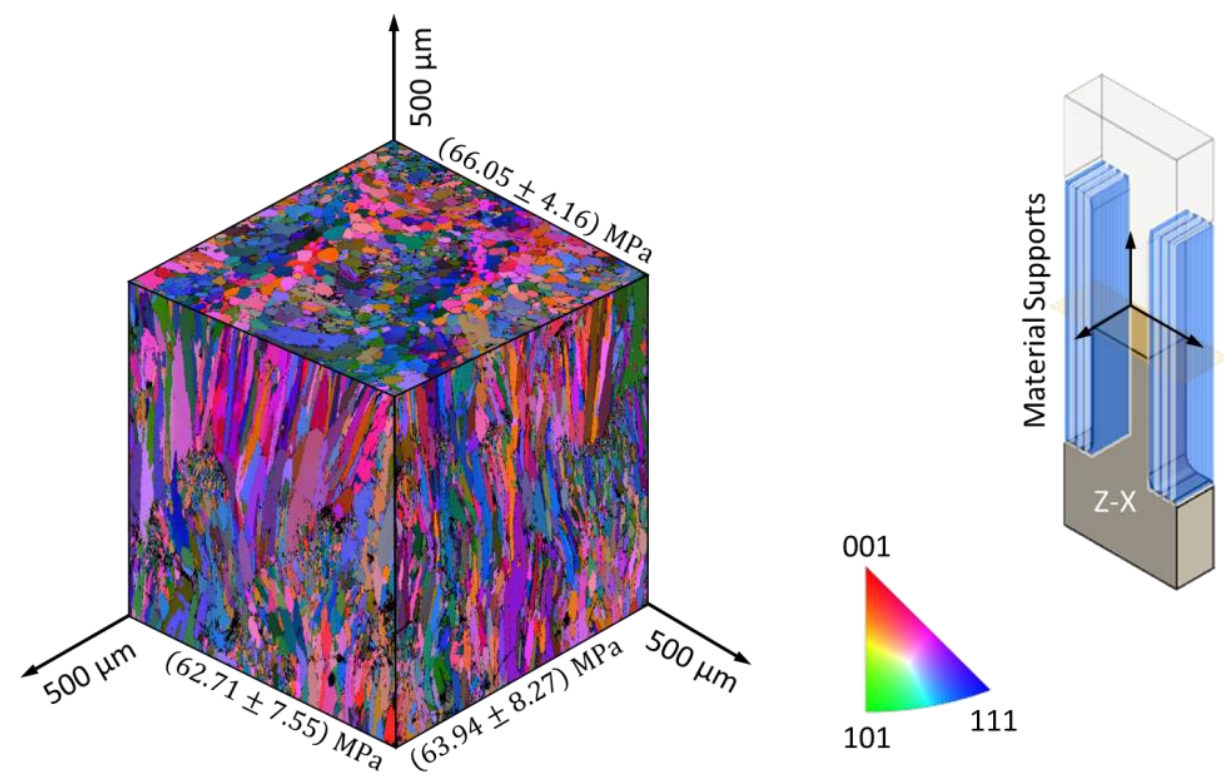

Figure 3. Grain pattern appearance in 3D-printed RS-333 Al alloy - ZX printing orientation. Youngs' modulus values derived from nanoindentation measurements are shown at respective planes.

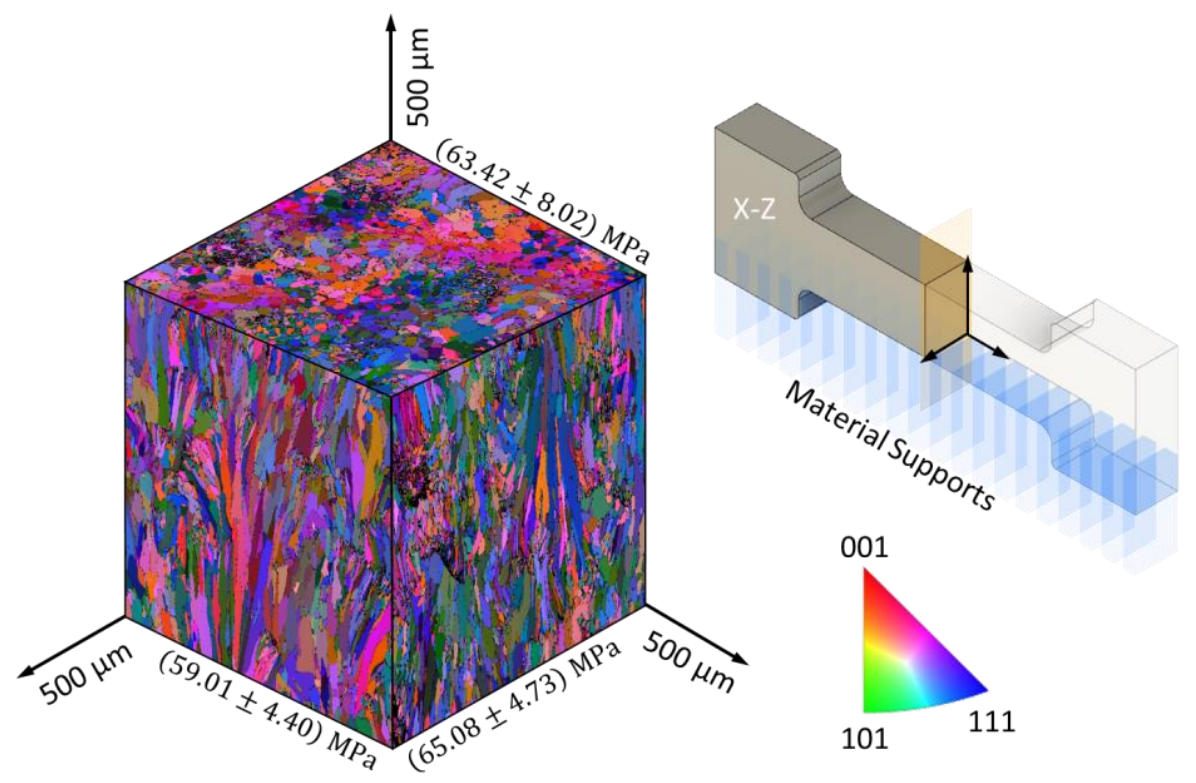

Figure 4. Grain pattern appearance in 3D-printed RS-333 Al alloy - XZ printing orientation. Youngs' modulus values derived from nanoindentation measurements are shown at respective planes.

Average values of grain dimensions for different printing orientations are presented in Table 1. Even qualitative analysis of grain patterns for different printing orientations gives opportunities to suggest that at least plasticity and fracture behavior at tension will be affected by columnar grains' orientation in respect of loading direction. In case of ZX 
printing orientation of dog bone sample columnar grains are parallel to tension axis. In opposite, for $\mathrm{XZ}$ and $\mathrm{XY}$ printing orientation columnar grains are perpendicular to tension axis making them structurally similar.

Table 1. Statistic information from EBSD analysis about length, width, and aspect ratio of grains for different specimen orientations.

\begin{tabular}{|c|c|c|c|c|c|c|c|c|c|}
\hline & \multicolumn{3}{|c|}{$\mathbf{Z X}$} & \multicolumn{3}{|c|}{$X Z$} & \multicolumn{3}{|c|}{$\mathbf{X Y}$} \\
\hline & $\begin{array}{c}\text { Length, } \\
\mu \mathrm{m}\end{array}$ & $\begin{array}{c}\text { Width, } \\
\mu \mathrm{m}\end{array}$ & $\begin{array}{l}\text { Aspect } \\
\text { ratio }\end{array}$ & $\begin{array}{c}\text { Length, } \\
\mu \mathrm{m}\end{array}$ & $\begin{array}{c}\text { Width, } \\
\mu \mathrm{m}\end{array}$ & $\begin{array}{c}\text { Aspect } \\
\text { ratio }\end{array}$ & $\begin{array}{c}\text { Length, } \\
\mu \mathrm{m}\end{array}$ & $\begin{array}{c}\text { Width, } \\
\mu \mathrm{m}\end{array}$ & $\begin{array}{c}\text { Aspect } \\
\text { ratio }\end{array}$ \\
\hline Surface & $19.7 \pm 19.3$ & $7.4 \pm 5.5$ & $2.5 \pm 1.3$ & $19.3 \pm 18.5$ & $7.5 \pm 5.6$ & $2.5 \pm 1.2$ & $22.3 \pm 17.1$ & $12.7 \pm 9.1$ & $1.8 \pm 0.5$ \\
\hline Side & $20.8 \pm 17.9$ & $7.6 \pm 5.2$ & $2.5 \pm 1.2$ & $11.5 \pm 7.2$ & $7.1 \pm 4.1$ & $1.6 \pm 0.4$ & $13.8 \pm 11.8$ & $6.4 \pm 3.6$ & $2.1 \pm 0.9$ \\
\hline Cross-section & $15.7 \pm 9.1$ & $9.4 \pm 4.8$ & $1.7 \pm 0.5$ & $22.0 \pm 18.4$ & $8.5 \pm 4.4$ & $2.5 \pm 1.2$ & $16.5 \pm 16.0$ & $6.3 \pm 4.0$ & $2.5 \pm 1.3$ \\
\hline
\end{tabular}

Although more focused analysis of grain size distributions is needed (as discussed round spot regions form a specific grain assembly) even the consideration of average grain sizes $D_{a v}=\sqrt{\text { Length } \times \text { Width }}$ can be useful.

Shear bands characteristic for ductile metals (and $\mathrm{Al}$ alloys) localize plastic flow in the directions forming an angle of about $45^{\circ}$ with tension axis. Thus, we suggest that different orientation of main axes of columnar grains in respect of shear band plane may take place. Therefore, the yield strength, that correlates (in accordance with Petch-Hall law) with mean crystallite size in the plane of shear band, is expected to be strongly dependent on orientation of columnar grains, i.e. printing orientation. For the simplicity, we estimate the average size of grain geometrical projection to the shear band plane by formula:

$$
D_{\text {av shear band }}=\sqrt{D_{\text {av surface }}^{2}+D_{\text {av cross-section }}^{2}}
$$

The values taken from Table 1 return the estimates for the average values of grain dimension projections to shear band plane $\left(45^{\circ}\right)$ as $17.3,18.3$ and $19.7 \mu \mathrm{m}$ for $\mathrm{ZX}, \mathrm{XZ}$ and $\mathrm{XY}$ respectively. This directly assumes that strength should scale down for $Z X, X Z$ and $X Y$ respectively.
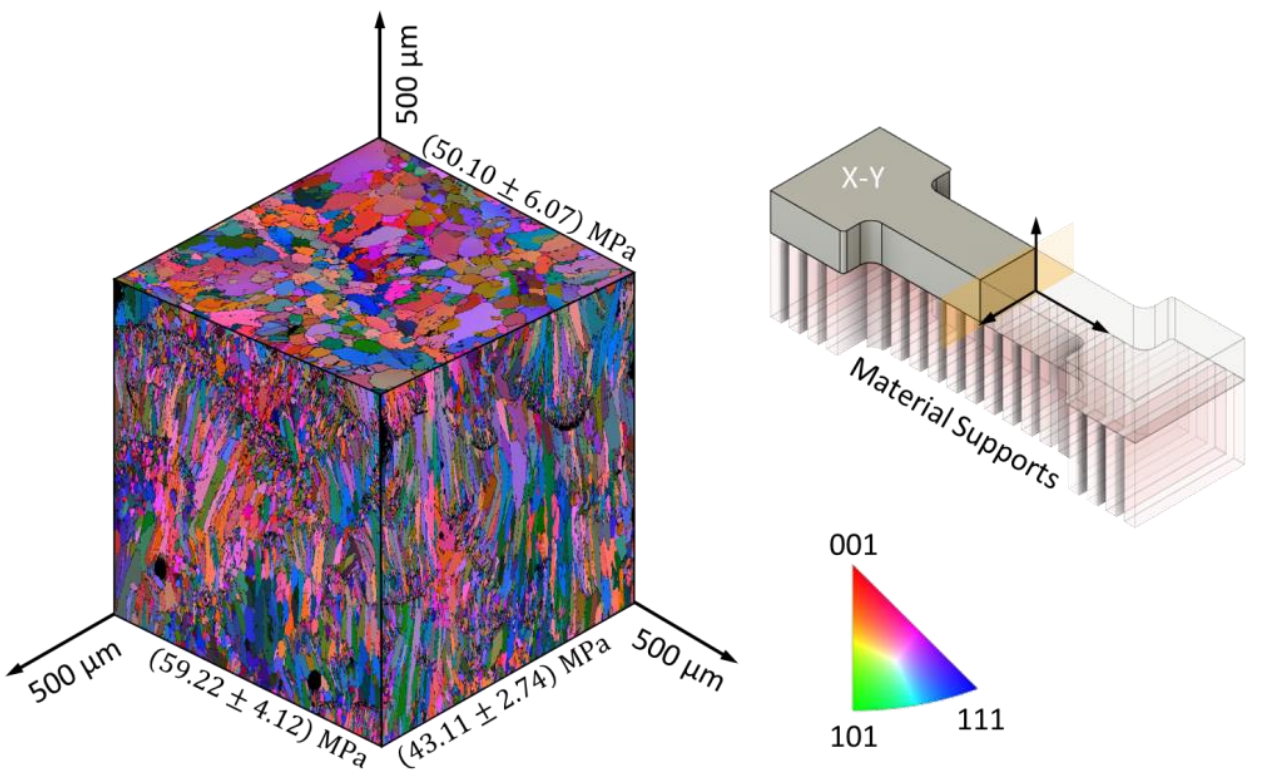

Figure 5. Grain pattern appearance in 3D-printed RS-333 Al alloy - XY printing orientation. Youngs' modulus values derived from nanoindentation measurements are shown at respective planes. 
Aluminum in contrast with some other FCC metals such as nickel is almost elastically isotropic. Therefore, the presence of texture in these 3D-printed Al alloy samples should not affect the overall elastic behavior.

On the other hand, we have shown recently [13] that apparent macroscopic mod-ulus noticeably varies (when calculated from in SEM tensile testing with strains measured using DIC of high-resolution images) for XY, XZ and ZX printing orientations - 65.4, 70.0 and $77.8 \mathrm{GPa}$, respectively.

Nanoindentation Oliver-Pharr tests after proper correction [15, 17] return reasonable values for the estimates of Young's modulus - see Figure 6 - and the orientation dependence of average values is in good accordance with previous report [13], namely for XY, XZ and ZX printing orientations we detected 50.8, 62.5 and 64.2 GPa, respectively. It is worth noting that the values of Young's modulus measured in the plane perpendicular to the main axis of the dog-bone sample gauge (cross-section), parallel to narrow (side) and wide (surface) faces of gauge zone, varies only slightly, showing almost no statistically significant distribution along the growth direction.

The dependence of elastic moduli on printing orientation is discussed in literature [19]. Since no crystallographic texture is detected in studied RS-333 one can suggest that chemical inhomogeneity, or preferential orientation of precipitates or porosity at nanometer dimensional scale (unresolved in SEM with magnification applied) may cause experimentally observed orientational dependence of Young's modulus motivating further fundamental research in this field with relevant methods - high resolution TEM, atom probe tomography, etc.

In contrast to obvious orientation anisotropy of Young's modulus it seems that the hardness is almost constant (around $0.8 \mathrm{GPa}$ ), isotropic and it has normal distribution with significance level 0.05 in all studied samples at many of studied planes (cross-section for $Z X$ and $X Z$; side for $Z X$ and surface $Z X)$. 

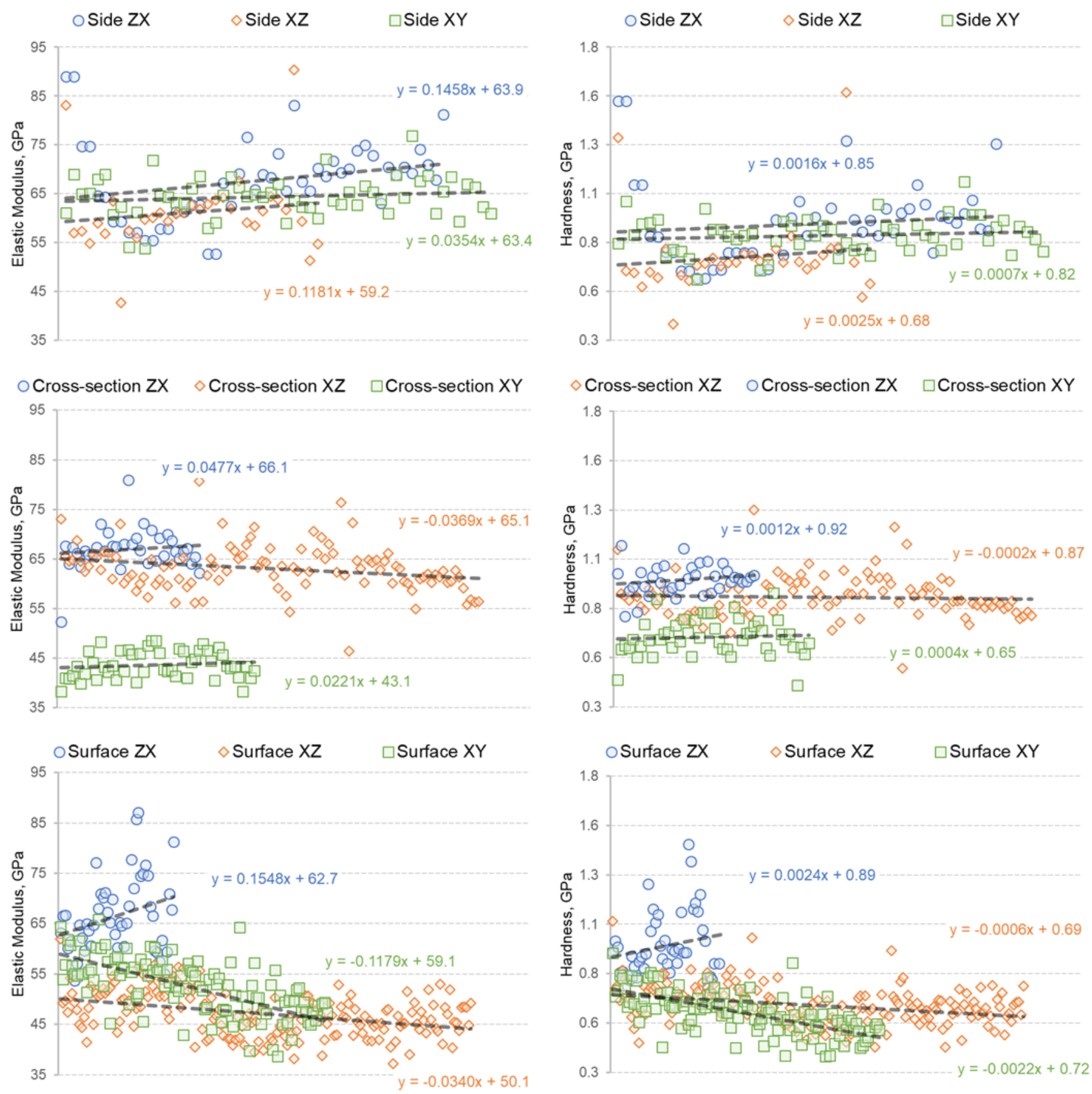

Figure 6. Young's modulus estimates (derived from Oliver-Pharr nanoindentation tests) in 3Dprinted RS-333 Al alloy.

Berkovich hardness value of about $0.7 \ldots 0.8 \mathrm{GPa}$ can be used for rough estimation of yield strength [20], that is approximately $1 / 3$ of hardness value - this characteristic is expected to be as high as $250 \mathrm{MPa}$. This value seems to be a good reference for Al-Mg-Si alloys (characteristic yield strength of artificially aged 6061 and 6156 wrought alloys is maximum 280 and $380 \mathrm{MPa}$ respectively) but not perfectly corresponds to 3D-printed RS$333 \mathrm{Al}$ alloy. In [13] we recently shown that yield strength at $0.2 \%$ of strain derived from tension stress-strain curve for 3D-printed RS-333 alloys varies for different printing orientations and reaches only $81 \mathrm{MPa}, 132 \mathrm{MPa}$ and $150 \mathrm{MPa}$ for $\mathrm{XY}, \mathrm{XZ}$ and ZX samples respectively. We suppose that the discrepancy be-tween values of yield strength derived from tensile test and nanoindentation may be related with strong influence of thin superficial surface layer on the results of measurements and therefore microhardness testing probing deeper regions of material is more suitable than nanoindentation for prompt and 
adequate characterization of mechanical response. On the other hand, the influence of porosity at nanometer dimensional scale may also contribute to the apparent deficiency of yield strength at tension, since, in contrast to tension, compression modes of plastic deformation tend to densify porous materials pushing up measured values of strength characteristics.

\subsection{Tensile test of 3D-printed RS-333 alloy. The influence of surface quality}

Stress-strain curves acquired in SE microscope are depicted in Figure 7 for the samples as 3D-printed in different printing orientations (as-printed), further mechanically polished with a series of grinding papers and diamond paste and, separately, sandblasted after 3D-printing. One can easily see that for as-printed samples ZX printing orientation arrives with favorable mechanical performance, i.e. combination of highest values of elastic modulus, ultimate tensile strength and large elongation till rupture. XZ orientation results in the highest ductility while $\mathrm{XY}$ orientation occurs to be the most brittle. Yield and tensile strength show practically the same dependence on printing orientations, that correlates with the results of nanoindentation testing.

The same general mechanical response for all printing orientations studied is inherited after mechanical polishing, bringing tensile strength characteristics and ductility to somewhat higher values and reducing, however, Young's modulus and yield strength. This fact supports the observation that surface layers may significantly affect mechanical response, since thermal history, heat fluxes and, perhaps, grain pattern are substantially different at the surface and in the core of 3D-printed samples. In [13] the residuals of supports, microcracks and traces of unmolten powder were reported as typical surface features. The volume of surface layer is of especially great importance for thin samples studied. We conclude that the removal of defect-rich surface layers improves tensile strength and ductility. The presence of tensile residual stresses in the superficial layer would reduce apparent yield strength and, perhaps, elongation till rupture. This issue is of our current interest and thorough investigations.

Sandblasting returns ambiguous results - it improves ductility of $X Y$ samples, but worsens ductility of $\mathrm{XZ}$ and $\mathrm{ZX}$ samples. Elastic and strength characteristics change in controversial direction suggesting that complex modification of superficial zone and residual stress re-distribution.

These results demonstrate that manipulations with 3D-printing orientation and relatively simple and commonly applied mechanical methods of surface treatment give wide range of opportunities for the fine tuning of mechanical response in structural elements of aerospace constructions to purposefully create high demanding parts by design.

Many practical issues, however, rest unanswered. As we demonstrated the columnar grains tend to be strictly aligned in growth direction, however, lys français and spot grain patterns often appear in some directions as well. The formation of these structures is to be thoroughly quantitatively studied and digitally modeled on a multiphysics platform that should create both fundamental theory of property moderation at SLM 3D printing and a practical tool (plug-in to CAD software) for the optimization of CAM printing code. 


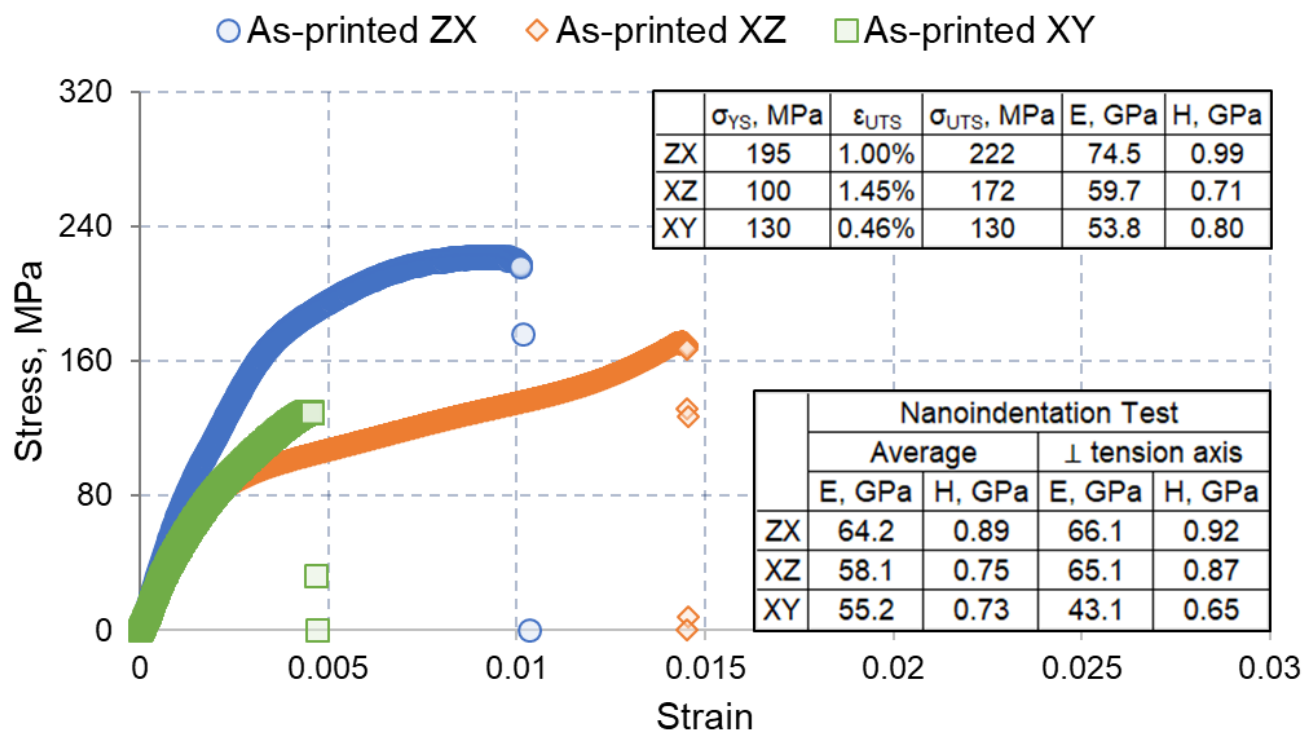

OPolished ZX $\diamond$ Polished XZ $\square$ Polished XY
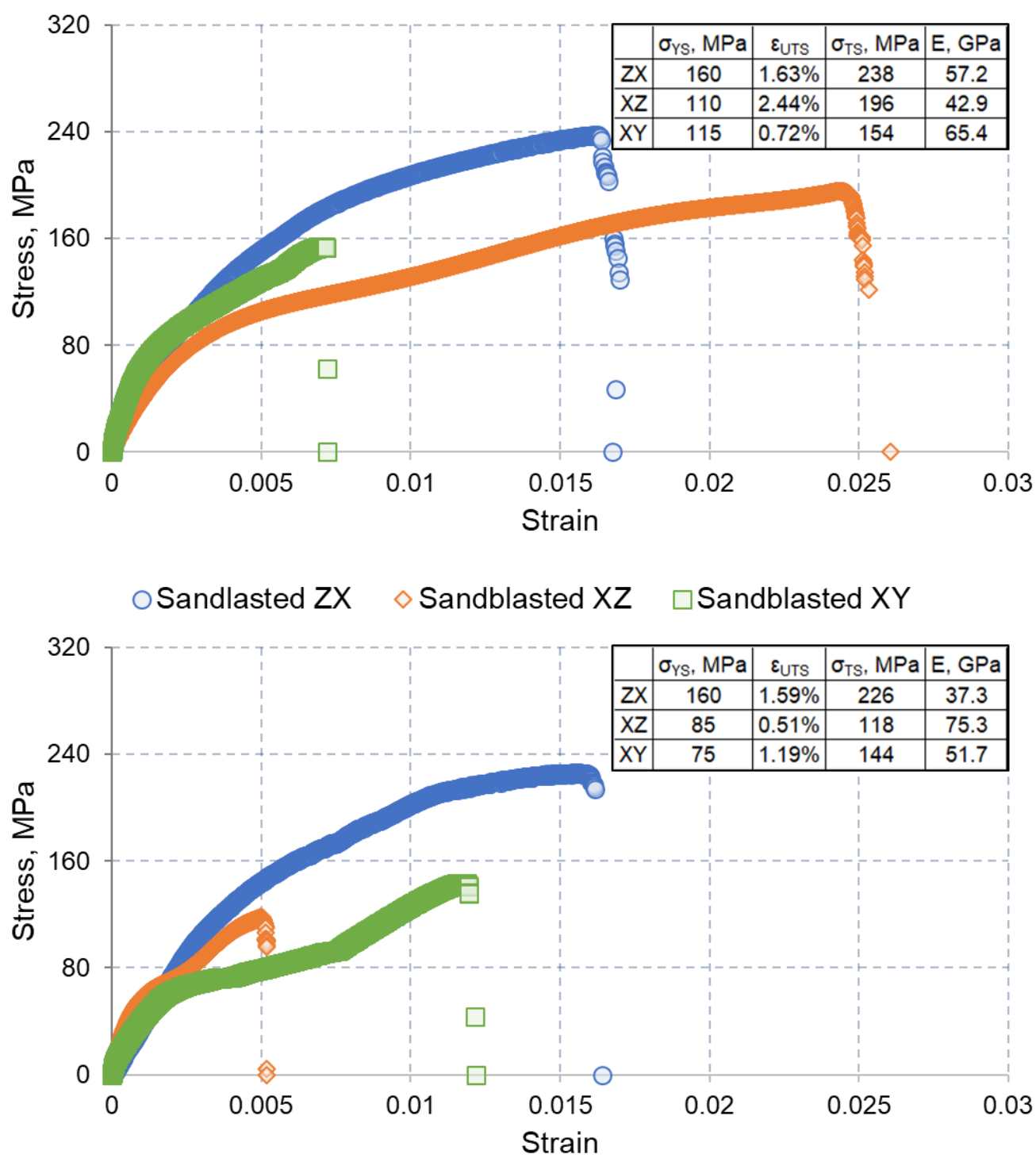

Figure 7. Mechanical response of 3D-printed RS-333 Al alloy under tension. 


\section{Conclusions}

3D-printed Al alloys rapidly fill the portfolio of efficient material solutions in aerospace giving chance for inexpensive fabrication of weight saving structural elements. Optimization of printing parameters allows to create specific patterns of columnar grains rationally oriented against load directions and through this to reinforce the parts in simple and efficient way. EBSD microscopy in combination with in SEM mechanical testing is very suitable tools for fundamental research in this field. As expected growing number of reports will appear in nearest future bridging gaps in understanding of grain patter formation and interpretation of anisotropy of elastic moduli in theoretically isotropic metals.

Additional phenomenon that ought to be taken into account concerns the introduction and presence of residual stresses within 3D-printed parts. Residual stresses appear as a consequence of the high temperature gradients and steep cooling rates during sample build that affect alloy ultra-structure (precipitates) and hence flow stress. This, in turn, gives rise to non-uniform plastic deformation and locked-in (or frozen in) permanent inelastic strains, often referred to as eigenstrains that can be thought of as the underlying origin of residual stress, and conversely, distortion.

The case of Aluminum alloys is of particular interest due to the near isotropy of their elastic properties that means that grain-level deformation-induced misfit strains arise only due to anisotropy of crystal plastic activity on the FCC $\{111\}<110>$ slip systems.

Residual stresses are known to exist over a variety of characteristic lengths and are of-ten classified into Type I (macro-), Type II (micro-) and Type III (nano-) scale [21,22]. Macroscopic Type I stresses can be evaluated using a number of mechanical sectioning techniques, such as the contour method, slitting, blind hole drilling etc. Meso- to microscale residual stresses of Type II are more difficult to quantify, although a range of methods have been developed based on laboratory $X$-ray and neutron diffraction that allow distinguishing between groups of grains that differ in terms of crystal lattice orientation. Finally, Type III residual stresses exist at the intra-granular scale, so that probing them requires suitably refined methods, such as micro-focus Laue X-ray diffraction, electron diffraction (HR-EBSD), or Focused Ion Beam methods such as micro-ring-core FIB-DIC [21-23].

It is important to note that residual stresses may exert an influence on the overall deformation behavior of polycrystalline assemblies. Micro-scale residual stresses have been shown to obey a statistical distribution that is well approximated by the Gaussian law $[21,24]$. This observation implies that some material volumes may find themselves close to the yield condition, causing plastic deformation to occur even at the early stages of loading. This may be understood e.g., through the use of Masing model [25] and lead to the modification of the apparent modulus upon loading.

The ultimate purpose of the analysis of the internal hierarchical residual stress state is to factor it into the prediction of material strength and failure, e.g., under the conditions of fatigue loading, as has been demonstrated for Ni-base superalloy samples produced by laser powder bed fusion (LPBF) [26]. The extension of this approach to different classes of metallic alloys remains an important theme of ongoing research.

Author Contributions: Conceptualization, A.I.S. and A.M.K.; methodology, Yu.M. and P.A.S.; software, E.S.S.; validation, A.I.S., D.R. and A.M.K.; formal analysis, E.S.S.; investigation, P.A.S. and E.S.S.; resources, A.I.S.; data curation, P.A.S. and E.S.S.; writing-original draft preparation, A.I.S. and E.S.S.; writing - review and editing, P.A.S., D.R. and A.M.K.; visualization, E.S.S. and P.A.S.; supervision, A.I.S., D.R. and A.M.K. All authors have read and agreed to the published version of the manuscript.

Funding: This research was funded by the Russian Science Foundation, grant number 21-19-00791, https://rscf.ru/en/project/21-19-00791/.

Data Availability Statement: The data presented in this study are available on request from the corresponding author. 
Acknowledgments: This research was funded by the Russian Science Foundation, grant number 21-19-00791, https://rscf.ru/en/project/21-19-00791/, and the authors are grateful to the crew of Fablab of Skoltech for delicate and accurate samples preparation.

Conflicts of Interest: The authors declare no conflict of interest.

1. Munir, Kh.; Biesiekierski, A.; Wen, C.; Li, Yu. Selective laser melting in biomedical manufacturing. Metallic Biomaterials Processing and Medical Device Manufacturing 2020, 235-269. https://doi.org/10.1016/B978-0-08-102965-7.00007-2

2. Zhang, L-Ch.; Wang, J.; Liu, Yu.; Jia, Zh.; Liang, Sh.-X. Additive Manufacturing of Titanium Alloys. Reference Module in Materials Science and Materials Engineering 2020. https://doi.org/10.1016/B978-0-12-819726-4.00002-8

3. Song, X.; Zhai, W.; Huang, R.; Fu, J.; Fu, M.; Li, F. Metal-Based 3D-Printed Micro Parts \& Structures. Reference Module in Materials Science and Materials Engineering 2020. https://doi.org/10.1016/B978-0-12-819726-4.00009-0

4. New nickel 3D printing powder ideal for gas turbine production. Available online: https://www.pesmedia.com/eos-nickeladditive-manufacturing-18022021/ (accessed on 6 June 2021).

5. Multi-metal 3D printed gas turbine. Available online: https://www.materialstoday.com/additive-manufacturing/products/multimetal-3d-printed-gas-turbine/ (accessed on 6 June 2021).

6. Satellite Detail Produced through 3D Printing by RUSAL Successfully Works in Spaceю Available online: https://www.spotlightmetal.com/satellite-detail-produced-through-3d-printing-by-rusal-successfully-works-in-space-a-998012/ (accessed on 6 June 2021).

7. Nguyen, D.-S.; Park, H.-S.; Lee, C.-M. Applying Selective Laser Melting to Join Al and Fe: An Investigation of Dissimilar Materials. Appl. Sci. 2019, 9, 3031. https://doi.org/10.3390/app9153031

8. Xiong, W.; Hao, L.; Li, Ya.; Tang, D.; Cui, Q.; Feng, Z.; Yan, Ch. Effect of selective laser melting parameters on morphology, microstructure, densification and mechanical properties of supersaturated silver alloy. Materials E Design 2019, 170. https://doi.org/10.1016/j.matdes.2019.107697.

9. Kluczyński, J.; Śnieżek, L.; Grzelak, K.; Janiszewski, J.; Płatek, P.; Torzewski, J.; Szachogłuchowicz, I.; Gocman, K. Influence of Selective Laser Melting Technological Parameters on the Mechanical Properties of Additively Manufactured Elements Using 316L Austenitic Steel. Materials 2020, 13, 1449. https://doi.org/10.20944/preprints202003.0015.v1

10. Pekok, M.A.; Setchi, R.; Ryan, M.; et al. Effect of process parameters on the microstructure and mechanical properties of AA2024 fabricated using selective laser melting. Int J Adv Manuf Technol 2021, 112, 175-192. https://doi.org/10.1007/s00170020-06346-y

11. Subbiah, R.; Bensingh, J.; Kader, A.; et al. Influence of printing parameters on structures, mechanical properties and surface characterization of aluminium alloy manufactured using selective laser melting. Int J Adv Manuf Technol 2020, 106, 5137-5147. https://doi.org/10.1007/s00170-020-04929-3

12. Koutny, D.; Skulina, D.; Pantělejev, L.; Paloušek, D.; Lenczowski, B.; et al. Processing of Al-Sc aluminum alloy using SLM technology. Procedia CIRP 2018, 74, 44-48. https://doi.org/10.1016/j.procir.2018.08.027

13. Statnik, E.S.; Nyaza, K.V.; Salimon, A.I.; Ryabov, D.; Korsunsky, A.M. In Situ SEM Study of the Micro-Mechanical Behaviour of 3D-Printed Aluminium Alloy. Technologies 2021, 9, 21. https://doi.org/10.3390/technologies9010021

14. Liu, X.; Zhao, C.; Zhou, X.; Shen, Z.; Liu, W. Microstructure of selective laser melted AlSi10Mg alloy. Materials $\mathcal{E}$ Design 2019, 168. https://doi.org/10.1016/j.matdes.2019.107677

15. Oliver, W.C.; Pharr, G.M. An improved technique for determining hardness and elastic modulus using load and displacement sensing indentation experiments. Journal of Materials Research 1992, 7, 1564-1583. https://doi.org/10.1557/JMR.1992.1564

16. Hay, J.C.; Bolshakov, A.; Pharr, G.M. A critical examination of the fundamental relations used in the analysis of nanoindentation data. Journal of Materials Research 1999, 14, 2296-2305. https://doi.org/10.1557/JMR.1999.0306

17. Yetna N'Jocka, M.; Roudet, F.; Idriss, M.; Bartier, O.; Chicot, D. Work-of-indentation coupled to contact stiffness for calculating elastic modulus by instrumented indentation. Mechanics of Materials 2016, 94, 170-179.

https://doi.org/10.1016/j.mechmat.2015.12.003

18. Blaber, J.; Adair, B.; Antoniou, A. Ncorr: Open-Source 2D Digital Image Correlation Matlab Software. Exp Mech 2015, 55, 1105-1122. https://doi.org/10.1007/s11340-015-0009-1

19. Ponnusamy, P.; Rahman Rashid, R.A.; Masood, S.H.; Ruan, D.; Palanisamy, S. Mechanical Properties of SLM-Printed Aluminium Alloys: A Review. Materials 2020, 13, 4301. https://doi.org/10.3390/ma13194301

20. Tiryakioğlu, M.; Robinson, J.C.; Salazar-Guapuriche, M.A.; Zhao, Y.Y.; Eason, P.D. Hardness-strength relationships in the aluminum alloy 7010. Materials Science and Engineering: A 2015, 631, 196-200. https://doi.org/10.1016/j.msea.2015.02.049

21. Salvati, E.; Korsunsky, A.M. An analysis of macro- and micro-scale residual stresses of Type I, II and III using FIBDIC

22. micro-ring-core milling and crystal plasticity FE modelling. Intl. J. Plasticity 2017, 98, 123-138. https://doi.org/10.1016/j.ijplas.2017.07.004

23. Everaerts, J.; Salvati, E.; Uzun, F.; Romano Brandt, L.; Zhang H.J.; Korsunsky, A.M. Separating macro- (Type I) and micro(Type II.III) residual stresses by ring-core FIB-DIC milling and eigenstrain modelling of a plastically bent titanium alloy bar. Acta Materialia 2018, 156, 43-51. https://doi.org/10.1016/j.actamat.2018.06.035

24. Salvati, E.; Romano Brandt, L.; Mughal, Z.; Sebastiani, M.; Korsunsky, A.M. Generalised residual stress depth profiling at the nanoscale using focused ion beam milling. J. Mech. Phys. Solids 2019, 125, 488-501. https://doi.org/10.1016/j.jmps.2019.01.007 
25. Chen, J.W.; Korsunsky, A.M. Why is local stress statistics normal, and strain lognormal? Materials E Design 2021, $198,109319$. https://doi.org/10.1016/j.matdes.2020.109319

26. Korsunsky, A.M. A Teaching Essay on Residual Stresses and Eigenstrains, 2017. Butterworth-Heinemann, ISBN: 9780128109908.

27. Salvati, E.; Lunt, A.J.G., Heason, C.P.; Baxter, G.J., Korsunsky, A.M. An analysis of fatigue failure mechanisms in an additively manufactured and shot-peened IN718 nickel superalloy. Materials \& Design 2020, 108605.

https://doi.org/10.1016/j.matdes.2020.108605 